\title{
The Novel Hepatokine Ectodysplasin A Is Increased in Obesity and Reduced after Liraglutide Management
}

\section{Zhensheng Cai}

Affiliated Hospital of Jiangsu University

\section{Xia Deng}

Affiliated Hospital of Jiangsu University

\section{Panpan Zhang}

Affiliated Hospital of Jiangsu University

\section{Zhicong Zhao}

Affiliated Hospital of Jiangsu University

\section{Chang Guo}

Affiliated Hospital of Jiangsu University

Chenxi Wang

Affiliated Hospital of Jiangsu University

Lian Li

Affiliated Hospital of Jiangsu University

Haoxiang Li

Affiliated Hospital of Jiangsu University

Tian Gu

Affiliated Hospital of Jiangsu University

Ling Yang

Affiliated Hospital of Jiangsu University

\section{Dong Wang}

Affiliated Hospital of Jiangsu University

\section{Li Zhao}

Affiliated Hospital of Jiangsu University

Guoyue Yuan ( $\nabla$ yuanguoyue@ujs.edu.cn )

Affiliated Hospital of Jiangsu University

\section{Research Article}

Keywords: Ectodysplasin A, obesity, glucagon-like peptide-1 
Posted Date: May 17th, 2021

DOI: https://doi.org/10.21203/rs.3.rs-508975/v1

License: (c) (i) This work is licensed under a Creative Commons Attribution 4.0 International License. Read Full License 


\section{Abstract}

Background Ectodysplasin A (EDA), a new hepatokine, has been recently characterized to play a role in liver lipid metabolism and insulin resistance, but its physiological role remains scarcely acquainted in obesity. This study was the first time to determine the level of serum EDA in obesity, and to assess change in the levels of EDA after weight loss in obese mice.

Methods We analyzed the serum concentrations of EDA by enzyme-linked immunosorbent assay (ELISA) in 60 subjects including 30 normal weight and 30 obesity. Male C57BL/6J mice were fed with high-fat diet and injected by liraglutide to reduce weight. AML12 cells were induced by palmitic acid and treated with liraglutide. Quantitative real-time PCR and Western blot analysis were conducted to evaluate the expression of EDA.

Results Serum EDA levels were significantly higher in obesity than in normal weight subjects. It was positively correlated with body mass index (BMI). More importantly, the mRNA and protein expression of EDA reduced after liraglutide management in vivo and in vitro.

Conclusions The level of EDA increased significantly in obesity and decreased significantly after weight loss. It is suggested that EDA may be a novel hepatokine associated with obesity-related metabolic diseases.

\section{Background}

With the improvement of people's living standards, the prevalence of metabolic diseases such as diabetes and obesity were increasing. Obesity, especially visceral obesity, not only affects body shape, but also is considered to be a key risk factor for insulin resistance, diabetes, fatty liver and even cardiovascular and cerebrovascular diseases [1]. The dyslipidemia plays an important role in this relationship. As the largest metabolic organ, liver plays an important role in lipid metabolism and is a very active site for lipid accumulation, storage and depletion [2,3]. Current studies have shown that obesity, insulin resistance, nonalcoholic fatty liver disease (NAFLD) and other metabolic abnormalities can lead to reprogramming of liver secretory group [4,5] ,affect the secretion of hepatokine [6-8], and thus influence the occurrence and development of metabolic diseases.

Hepatokines, mainly secreted by the liver tissue, play a role through endocrine and paracrine pathways, and affect energy metabolism of liver and other organs through tissue communication $[6,9]$.

Ectodysplasin A (EDA), a newly discovered hepatokine, was thought to be associated with obesity, type 2 diabetes mellitus (T2DM), insulin resistance and NAFLD [10,11]. In vivo, overexpression of EDA can lead to impaired glucose tolerance induced by high-fat diet (HFD), while knockout of EDA can significantly reduce the formation of liver lipid droplets, the content of liver triglyceride (TG) and the levels of alanine aminotransferase (ALT) and aspartate aminotransferase (AST) in peripheral blood of obese mice induced by HFD [10]. In addition, EDA may promote the accumulation of TG in liver by influencing de novo synthesis of fat and TG lipolysis/fatty acid oxidation. The clinical research showed that circulating EDA 
levels were elevated in patients with NAFLD and the expression of it in liver tissue was positively correlated with visceral fat area, liver fat content and nonalcoholic steatohepatitis (NASH) scores [11]. A study from Awazawa and his coworkers in 2017 indicated that the EDA of 23 obese subjects decreased significantly, and the body weight and insulin sensitivity were significantly improved after surgical weight loss [10].

Glucagon-like peptide-1 receptor agonists (GLP-1 RAs) are a new class of pharmacological agents that improve glucose homeostasis in many ways [12], however, clinical studies have also shown that they can reduce the body weight and improve the blood lipid profile of patients with T2DM $[13,14]$ and ameliorate nonalcoholic hepatic steatosis and nonalcoholic steatohepatitis $[15,16]$. Liraglutide is the most recent GLP-1 RA to be approved for weight loss by the U.S. Food and Drug Administration [17]. A multi-center, double-blinded, randomized, placebo-controlled trial proved that liraglutide was safe, well tolerated, and led to histological resolution of non-alcoholic steatohepatitis [18]. Therefore, the aim of this study was to explore the association between the hepatokine EDA and obesity and the change of EDA after weight loss and liver function improvement.

\section{Methods}

\subsection{Study participants}

All participants were recruited at the Affiliated Hospital of Jiangsu University, Jiangsu, China. Approval for the study was obtained from the Clinical Research Ethics Committee, Affiliated Hospital of Jiangsu University. All methods were carried out in accordance with relevant guidelines and regulations. A total of 60 age- and sex-matched participants were recruited for this study. Based on body mass index (BMI), all subjects were divided into 2 subgroups: normal-weight (NW: BMl $25 \mathrm{~kg} / \mathrm{m}^{2}, \mathrm{n}=30$ ) and obesity(OB: $\mathrm{BMl} \geq 25 \mathrm{~kg} / \mathrm{m}^{2}, \mathrm{n}=30$ ) [19]. In this study, we excluded subjects with any of the following: acute complications of diabetes, secondary obesity, acute and chronic inflammatory diseases, systemic corticosteroid treatment, renal dysfunction, or hepatic dysfunction. Women who were currently pregnant, breastfeeding, or taking a contraceptive pill were also excluded from the study.

\subsection{Measurement of EDA concentrations and biochemical parameters}

Serum EDA levels were determined using a commercially available human enzyme-linked immunosorbent assay (ELISA) (Wuhan Eiaab Science Co., China; Catalog No. E1976h). The sensitivity of the kit was less than $20 \mathrm{pg} / \mathrm{ml}$, the intra assay CV was $\leq 7.8 \%$, and the inter assay CV was $\leq 8.9 \%$. The detection range of ELISA was $78-5000 \mathrm{pg} / \mathrm{mL}$.

Clinical and biochemical evaluations were performed as described previously [20]. Height and weight were measured using standardized techniques. BMI was calculated as body weight ( $\mathrm{kg}$ ) divided by square of height $(\mathrm{m})$ and the waist-to-hip ratio (WHR) was calculated by the ratio of waist circumference (WC) to hip circumference (HC). Participants were told to avoid stressful activities (sports and physical exercise) before blood collection. Blood samples were obtained from each subject after overnight fasting. 
Serum samples were separated and not frozen for biochemical analysis. Fasting plasma glucose (FPG) was determined by a glucose oxidase-based assay and fasting insulin (FINS) was measured by chemiluminescence. Hemoglobin A 1c ( HbA1c) was determined using HPLC. The levels of TG, total cholesterol (TC), high density lipoprotein cholesterol (HDL-C), low density lipoprotein cholesterol (LDL-C), ALT and AST were detected by enzymatic method and glutamyl transpeptidase (GGT) was determined by $y$-glutamine-3-carboxyl-4-nitroaniline method. To estimate insulin sensitivity and $\beta$-cell function, the homeostasis model assessment (HOMA) was used: HOMA of insulin resistance (HOMA-IR) $=$ FINS (milliunits per liter) $\times F P G$ (millimoles per liter)/22.5, HOMA of $\beta$-cell function (HOMA- $\beta)=20 \times F I N S /(F P G-$ 3.5).

\subsection{Animals and experimental design}

Twenty-four healthy male C57BL/6J mice, four weeks of age, were maintained in a room with controlled lighting (12 hours light/dark cycle) and regulated temperature $\left(23 \pm 2^{\circ} \mathrm{C}\right)$ and humidity $(50-60 \%)$. All mice were fed with free access to food and water for 1 week to be adapted for the environment. After 1 week of acclimation, eight mice were randomly fed with regular chow as normal control group (NC group) and throughout the study, while the remaining mice were fed with HFD (HFD: $36 \%$ carbohydrate, $19 \%$ protein and $45 \%$ fat) for 20 weeks. Then, the HFD animals were randomly divided into two groups: HFD+saline group (HFD group), or liraglutide (LIRA, Victoza, Novo Nordisk)-treated group (HFD+LIRA group). They were received daily subcutaneous injections with either liraglutide $(0.2 \mathrm{mg} / \mathrm{kg}$ daily) or the same volume of saline at about $12.30 \mathrm{pm}$ for 12 weeks and normal chow mice were given saline injections during the same period. The body weight of all mice measured every 2 weeks.

At the end of the experiment, the mice were fasted overnight and euthanized using $1 \%$ sodium pentobarbital $(50 \mathrm{mg} / \mathrm{kg}$ ). Eyeballs were removed and blood samples were collected and serum was obtained by centrifugation at $3,000 \mathrm{rpm}$ at $4^{\circ} \mathrm{C}$ for $20 \mathrm{~min}$. Tissues collected were either immediately fresh frozen in liquid nitrogen after dissection and stored at $-80{ }^{\circ} \mathrm{C}$ until further processing or were fixed in $4 \%$ paraformaldehyde (PFA) solution in PBS.

\subsection{Cell culture and insulin resistance models}

Alpha mouse liver 12 (AML12) cells were obtained from cell bank of Chinese Academy of Science, Shanghai., and maintained in Dulbecco's modified Eagle's medium (DMEM) (Gibco) with $25 \mathrm{mM}$ glucose, 10\% fetal bovine serum (FBS) (Gibco), penicillin (100 units $/ \mathrm{ml})$, streptomycin (100 $\mu \mathrm{g} / \mathrm{ml})$ (Sigma), insulin, transferrin and selenium (ITS) (Sigma) and dexamethasone $(40 \mathrm{ng} / \mathrm{ml})\left(\right.$ Sigma) at $37^{\circ} \mathrm{C}$ in a humidified atmosphere of $95 \%$ air and $5 \% \mathrm{CO}_{2}$. To induce insulin resistance and lipid metabolism disorder, AML12 cells were incubated with $0.25 \mathrm{mM}$ palmitic acid (PA) with $0.2 \%$ bovine serum albuminin (BSA) for $24 \mathrm{~h}$ in serum-free medium and cells were treated with different concentrations of liraglutide $(10 \mathrm{nM}, 100 \mathrm{nM}, 1000 \mathrm{nM})$ in the presence of PA.

\subsection{Biochemical analyses of animals' serum}


Serum blood glucose, TC, TG, LDL-C, as well as AST and ALT activities were examined by sop of Beckman Coulter Biochemical Analyzer (AU5800, USA).

\subsection{Insulin tolerance test and glucose tolerance test}

The insulin tolerance test (ITT) and glucose tolerance test (GTT) were performed as previously described $[21,22]$. Briefly, in ITT and GTT, mice were starved for $4 \mathrm{~h}$ and $16 \mathrm{~h}$ respectively, and then insulin $(0.75$ units $/ \mathrm{kg}$ ) or glucose $(2.0 \mathrm{~g} / \mathrm{kg}$ ) were injected intraperitoneally. Accu-check glucometer (Sandhofer Strasse 116,68305 Mannheim, Germany) was used to measure the blood glucose levels in the tail vein. Blood glucose was measured before injection (time 0 ) and at 15, 30, 60, 90, 120 min after injection in ITT, and before injection (time 0 ) and at 30, 60, 90, 120 min after injection in GTT.

\subsection{Tissue histology and liver triglyceride assay}

The sections of liver tissues were fixed in 4\% PFA, embedded in paraffin and sliced (4 $\mu$ m). Hematoxylin and eosin (HE) staining was performed according to the standard procedure, and imaged under a light microscope (Nikon, Japan). Hepatic lipid accumulation was also determined using Oil Red 0 staining. The imaging system was used to collect the images on the tissue staining section, and the analysis software was used to automatically read the tissue measurement area, calculate the positive area and tissue area in the measurement area, and calculate the proportion of the positive area (Servicebio, China). Intrahepatic (mice) TGs were measured using a TG assay kit (Jiancheng Bioengineering Institute, Nanjing, China).

\subsection{Quantitative real-time PCR}

Total RNA was isolated from cells or livers using TRIzol Reagent (Invitrogen) followed by the manufacturer's guidelines and cDNA was generated with PrimeScript RT-PCR Kit (Vazyme Biotech, Nanjing, China). PrimeScript RT-PCR Kit (Vazyme Biotech, Nanjing, China) was used for measuring relative mRNA expression by quantitative real-time PCR (Quant Studio 5). EDA gene expression levels were normalized to $\beta$-actin levels. The sequences of primers (Sangon Biotech, Shanghai, China) used in the study were listed as followed: EDA, forward TGAATAGCAGCCCATTAGTAGG and reverse CAGAGAATAAATGGCATTGGCA; $\beta$-actin, forward TGGAATCCTGTGGCATCCATGAAAC and reverse TAAAACGCAGCTCAGTAACAGTCCG.

\subsection{Western blot analysis}

Total proteins were extracted from livers and cells by with ice-cold RIPA lysis buffer (Beyotime, Shanghai, China) and quantified with BCA kits (Beyotime, Shanghai, China). The denatured protein was loaded and separated on a $10 \%$ SDS-PAGE gel, transferred onto PVDF membranes, and then blocked with $5 \%$ non-fat milk in Tris-buffered saline for $1 \mathrm{~h}$. Subsequently, the membranes were incubated with the EDA (Abcam) and $\beta$-actin (CST) primary antibody overnight at $4^{\circ} \mathrm{C}$. The following day, appropriate secondary antibodies conjugated to horseradish peroxidase were incubated with respective membranes for $1 \mathrm{~h}$ at room temperature. The bands were detected by enhanced chemiluminescence $(E C L)$ method using kit (Vazyme 
Biotech, Nanjing, China). The relative expression of target protein was normalized to that of $\beta$-actin. Protein band densities were quantified using ImageJ.

\subsection{Statistical analysis}

All statistical analyses were performed using SPSS 25.0. Data are presented as mean \pm standard deviation $(x \pm S D)$ for normally distributed variables and percentage $(n \%)$ for categorical variables. Data were tested for normality before the use of a parametric test. Independent student $t$ test was used for comparison between the two groups. One-way ANOVA were used for multiple comparisons. Categorical variables were compared by $\chi 2$ test. Relationships between EDA and other parameters were examined by calculation of Pearson correlation coefficients and Spearman correlation coefficients. Multivariate regression models were fit for EDA as a dependent variable to demonstrate the relative contribution of these parameters to the outcome ones after collinearity diagnostics. Drawing with software of GraphPad Prism 9.0 (GraphPad software, Inc., La Jolla, CA, USA). P<0.05 was considered significant.

\section{Results}

\subsection{Clinical and biochemical characteristics among groups}

The basic clinical and biochemical parameters of subjects were summarized and presented in Table 1. There was no statistically significant difference in age, sex, smoking status, alcohol consumption, HbA1c, FPG, TC, LDL-C, AST and $y-G T$ between the two groups. Compared with the NW group, BMI, WC, HC, WHR, SBP, DBP, FINS, TG, ALT, HOMA-IR and HOMA- $\beta$ in OB group were significantly increased $(P<0.05$ or $P<0.01)$, while HDL-C were largely decreased $(P<0.01)$. Serum EDA level was higher in OB than NW group $(P<0.001)$.

\subsection{Clinical and biochemical characteristics of the study objects according to the tertiles of EDA}

Subjects in the upper and middle serum EDA tertile had higher BMI, WC, HC, WHR and SBP compared with subjects in the lower serum EDA tertile $(P<0.05$ or $P<0.01)$. HDL-C was lower in the upper tertile group than in the lower tertile group (Table 2, $\mathrm{P}<0.05$ ). Other metabolic parameter such as TG, FINS, HOMA-IR and HOMA- $\beta$ were higher in the upper tertile group than in the middle one, although the differences did not reach statistical significance.

\subsection{Relationships between serum EDA levels and metabolic parameters}

Serum EDA concentrations were positively correlated with BMI, WC, HC, WHR and SBP but inversely correlated with AST (Fig.1). Multivariable linear regression models revealed that BMI was independently related to the serum EDA levels (Table3).

\subsection{Liraglutide induced body weight and liver weight loss}


The body weight of mice throughout the study was shown in Figure 2. At the beginning of the study, there was no significant difference in body weight between NC group and HFD group. At the 20th week, the weight of mice in HFD group was increased obviously compared to that of NC group, and the difference was statistically significant (Fig. $2 \mathrm{~A}, \mathrm{P}<0.05$ ). It is suggested that the model of obesity mice fed with HFD was successfully established. After intervention with liraglutide, the body weight of mice in HFD+LIRA group was significantly lower than that in HFD group (Fig. 2B, P<0.01). We also found that the HFD group showed clear increase in liver weight compared with NC group, and liraglutide treatment markedly reduced liver weight compared with HFD group (Fig. 2C).

\subsection{Liraglutide increased insulin sensitivity in HFD mice}

After 12 weeks of treatment, GTT and ITT were performed. After administration of glucose, there was no significant difference in blood glucose level and the area under the curve (AUC) of GTT between HFD+LIRA group and HFD group (Fig. 3A, B). What's more, the blood glucose level and AUC of ITT in HFD+LIRA group were significantly lower than those in HFD group after insulin administration (Fig. 3C, D, $P<0.01)$. These results suggested that liraglutide treatment could promote insulin hypoglycemic effect and improved HFD-induced insulin resistance.

\subsection{Liraglutide improved hepatic steatosis and reduced blood lipid levels}

In order to determine whether liraglutide could affect blood lipid levels, we further analyzed the lipid profile. The serum levels of TC, TG, LDL-C and ALT in HFD group were significantly higher than those in NC group (Fig. 4A, B, C, D, P<0.05), but the level of AST had no significant difference (Fig. 4E). After 12 weeks of liraglutide treatment, the results showed that the levels of TC, TG, LDL-C and ALT in HFD+LIRA group were significantly decreased compared to that HFD group (Fig. $4 A, B, C, D, P<0.05$ ). Then we investigated the effect of liraglutide on hepatic steatosis. By no accident, HE staining of liver tissue was consistent with lipid profile and the liver appearance was shown in Figure 5A. The ratios of the Oil Red Ostained area to the total area in the liver were significantly lower in the HFD+LIRA group than in the HFD group (Fig. 5C, $\mathrm{P}<0.01$ ). Although there was no difference between NC group and HFD group, the hepatic lipid accumulation in the HFD mice was more severe than that in the NC group in Figure 5B. In the HFD group, most of the hepatocytes showed vesicular degeneration and steatosis, and inflammatory cell infiltration was obvious. After liraglutide treatment, the hepatic lipid accumulation and liver steatosis were drastically improved. Also, mice fed 20 weeks of a HFD had significantly higher TG contents, liraglutide significantly attenuated the intrahepatic TG contents (Fig. 5D, $P<0.01$ ).

\subsection{The expression of EDA decreased after liraglutide treatment in HFD mice}

To explore whether the level of EDA has changed after liver lipid metabolism improvement, we analyzed the mRNA and protein expression of it in liraglutide treated obese mice. The relative mRNA expression level of EDA in HFD mice was increased significantly than that of NC group (Fig. 6A, $P<0.05$ ). In accordance with these observations, Western blot assessments revealed greater EDA protein expression in liver tissues from HFD-fed obese mice than their controls (Fig. 6B). Further studies showed that the 
mRNA and protein levels of EDA in HFD+LIRA group were significantly decreased than those in HFD group (Fig. $6 A, B, P<0.05$ or $P<0.01$ ). These results suggested that the expression of EDA decreased after liraglutide intervention in vivo.

\subsection{The expression of EDA decreased after liraglutide treatment in AML12 cells}

To investigate whether there existed similar results in vitro, we next analyzed the EDA expression in PAinduced AML12 cells. Compared with the control group, the mRNA and protein expression levels of EDA in PA group were significantly increased, while the mRNA and protein expression levels of EDA reduced significantly after liraglutide management in a dose-dependent manner (Fig. 7A, $B, P<0.05$ or $P<0.01$ ).

\section{Discussion}

In recent years, hepatokines have been identified and examined for their role in the development of obesity, NAFLD and insulin resistance [23]. The present study confirmed the association between a novel hepatokine EDA and obesity. We found that serum EDA level was higher in obesity subjects $\left(B M l \geq 25 \mathrm{~kg} / \mathrm{m}^{2}, n=30\right)$ than normal weight group $\left(B M l<25 \mathrm{~kg} / \mathrm{m}^{2}, n=30\right)$. There was also a positive correlation between EDA and BMI and WHR. More importantly, our experiment showed that the expression of EDA was elevated in HFD-fed obese mice and was decreased after liraglutide reduced body weight and improved hepatic lipid homeostasis. Similar to our results, 23 obese patients who underwent a two-step bariatric surgery strategy had lower liver EDA expression 12 months after the first surgery in parallel with reduction in body weight and improvement to insulin sensitivity [10].

In this study, we provide the first clinical evidence showing that serum levels of EDA, which has been suggested as a potential candidate for the treatment of NAFLD, are increased in obesity. Our results have demonstrated several parameters of adiposity (BMI, WC, $\mathrm{HC}, W H C$ ), insulin sensitivity and $\beta$-cell function (FINS, HOMA-IR and HOMA- $\beta$ ), lipid profiles (TG) and liver enzyme (ALT) were higher while HDL-C was decreased in OB subjects, indicating that obesity caused the glucose metabolism disorder and liver function abnormality. Furthermore, subjects in the upper EDA tertile had higher levels of BMI, WC, HC, WHR, and lower HDL than subjects in the lower terile. Other parameters of glucose metabolism and insulin function such as FINS, HOMA-IR and HOMA- $\beta$ were higher in the upper tertile group than in the middle one, although there was no statistical significance. Additionally, our study revealed a positive association between circulating EDA and BMI, WC, HC, WHR and a reverse with AST, probably because a relatively small sample size, different populations and different disease states. More importantly, BMI was independently related to the serum EDA levels, which demonstrated that BMI was a potential independent predictor of EDA. Recently, Awazawa et al. also found that in obese men ranging in BMl from 23 to $46 \mathrm{~kg} / \mathrm{m}^{2}$, hepatic EDA expression increased and correlated with visceral fat area, liver fat content, insulin resistance and NASH scores, however it reversed as weight loss [10]. Consistent with our results, others found significantly positive associations of EDA with a series of anthropometric parameters, e.g. age, BMI and WHR. However, EDA also correlated with other metabolic parameters such as FPG, HbA1c, HOMA-IR, but no parameter of liver enzymes [11], which was different with our results. These differences 
may be due to sample size and selected subjects. In our study, we enrolled obese patients but others selected NAFLD cases. Considering the correlation between EDA and BMI and WHR, we reasonably believe that EDA might be a serum biomarker and a potential therapeutic target for obesity.

Similarly, we have found that EDA was associated with obesity in experiments in vivo and in vitro. Obesity is characterized by adipocyte dysfunction including dyslipidemia [24], which is the main cause of insulin resistance and the increasing global obesity prevalence is closely coupled to the parallel increase in global rates of T2DM [25]. Many studies have shown that mice fed with HFD develop insulin resistance and obesity [23]. In the current study, HFD-fed mice also showed obesity, hyperlipidemia, insulin resistance and liver fat accumulation. Body weight and liver weight were elevated in mice after HFD treatment for 20 weeks. Moreover, HFD contributed to the increase in some lipid profiles such as TC, TG, LDL-C. The liver enzyme ALT was higher in HFD-fed mice and most of the hepatocytes showed vesicular degeneration and steatosis, suggesting that the liver function was impaired by HFD. Our research revealed that the EDA content was higher in mice after HFD treatment than that in control group. Notably, the relative mRNA and protein expression of EDA was also elevated in PA-induced AML12 cells with insulin resistance, which has not been discussed before, suggesting the potential relationship between EDA and insulin sensitivity in AML12 cells with lipid metabolism disorder. These results suggest that EDA may play a detrimental role in the occurrence and development of obesity, insulin resistance, and hepatic steatosis. Awazawa et al. overexpressed EDA in the livers of mice via adeno-associated virus (AAV)mediated gene transfer. In a glucose tolerance test, mice injected with EDA-AAV exhibited higher glucose concentrations and a decreasing tendency in energy expenditure than control GFP-AAV-injected mice [10]. In addition, another study showed that EDA knockdown attenuated hepatic lipogenesis via mediating lipolytic and lipogenic genes in vivo and in vitro. They found that expression of the key fatty acid oxidative enzyme carnitine palmitoyl transferase 1a (CPT1a) were significantly elevated in EDAknockdown HepG2 cell [11]. Previous studies and our results all indicated that EDA is associated with obesity, hyperlipidemia, abnormal liver function and insulin resistance.

It is known that GLP-1 RAs can improve glucose and cause weight loss, so they are receiving increasing attention for the treatment of diabetes-obesity. Recently, GLP-1 RAs have been used as a preferred treatment for patients with diabetes and obesity $[26,27]$. Previous studies have suggested that liraglutide can play a hypoglycemic role by increasing insulin secretion, improving islet cell function and reducing body weight in diabetic mice and obese rats [28-30]. Others also confirmed that GLP-1 RAs inhibited the formation of lipid droplets in different cells lines [31, 32]. Our results also showed that liraglutide promoted weight loss and improved insulin sensitivity in HFD-fed mice. What's more, our data suggested that liraglutide can alter the lipid profile by reducing serum TC and TG levels in HFD-fed mice. At the same time, it also significantly reduced the liver weight and hepatic steatosis of HFD-induced obese mice. Although the ratios of the Oil Red O-stained area to the total area had no statistical significance after HFD feeding, the hepatic lipid accumulation in images was more severe in HFD mice compared to NC group, probably because the large intra-group differences. More interestingly, we found that the expression of EDA was reduced significantly in the liver of HFD-fed mice and in AML-12 cells induced by PA after 
liraglutide treatment, suggesting that when obesity, insulin resistance and liver function were improved, the level of EDA was also decreased accordingly.

Based on our results, EDA may play a role in the development of obesity. However, there are several limitations in our study, including a relatively small sample size, limited population races and inadequate mechanism exploration. Our sample size is only 60 and limited to the Chinese population, which was not representative of the general population. In addition, our study design was cross-sectional and did not address the cause-effect relationship between serum EDA and obesity-related medical disorders. Also, in experiments in vivo and in vitro, there was no further study on the pathway mechanism of the relationship between EDA and obesity. Thus, additional studies are required to provide new insights into this subject.

\section{Conclusions}

In a whole, this study found that elevated circulating EDA levels were associated with obesity and mRNA and protein expression of it was progressively increased in liver tissue of obese mice and PA-induced hepatic cells, however it was decreased when liraglutide, a GLP-1 RA, reduced weight and improved lipid metabolism as well as insulin sensitivity in vivo and in vitro. This study for the first time demonstrated that EDA might be a potential biomarker for obesity and obesity-related glycolipid metabolism abnormity, but the deep mechanisms and causality are still largely unknown and need further researches.

\section{List Of Abbreviations}

AAV, adeno-associated virus; ALT, alanine aminotransferase; AML12, alpha mouse liver 12; AST, aspartate aminotransferase; BMI, body mass index; BSA, bovine serum albuminin; CPT1a, carnitine palmitoyl transferase 1a; DMEM, Dulbecco's modified Eagle's medium; ECL, enhanced chemiluminescence; EDA, Ectodysplasin A; ELISA, enzyme-linked immunosorbent assay; FINS, fasting insulin; FPG, fasting plasma glucose; GGT, glutamyl transpeptidase; GLP-1 RAs, Glucagon-like peptide-1 receptor agonists; GTT, glucose tolerance test; $\mathrm{HbA1c}$, hemoglobin A 1c; HC, hip circumference, $\mathrm{HDL}-\mathrm{C}$, high density lipoprotein cholesterol; HE, hematoxylin and eosin; HFD, high-fat diet; HOMA, homeostasis model assessment; HOMA-IR, HOMA of insulin resistance; HOMA- $\beta$, HOMA of $\beta$-cell function; ITS, insulin, transferrin and selenium; ITT, insulin tolerance test; LDL-C, low density lipoprotein cholesterol; NADH, nonalcoholic steatohepatitis; NAFLD, nonalcoholic fatty liver disease; NC, normal control; NW, normal-weight; OB, obesity; PA, palmitic acid; PFA, paraformaldehyde; T2DM, type 2 diabetes mellitus; TC, total cholesterol; TG, liver triglyceride; WC, waist circumference; WHR, waist-to-hip ratio.

\section{Declarations}

\section{Ethics approval and consent to participate}

Approval for the study was obtained from the Clinical Research Ethics Committee, Affiliated Hospital of Jiangsu University. All subjects signed informed consent forms. All animal experiments were in 
accordance with the guidelines and policies formulated by the Animal Care Committee of Jiangsu University experimental animal center. The study was carried out in compliance with the ARRIVE guidelines.

\section{Consent for publication}

Not Applicable.

\section{Competing interest}

The authors declare that they have no conflicts of interest associated with this article. No benefits in any form have been received or will be received from a commercial party related directly or indirectly to the subject of this paper.

\section{Availability of data and materials}

The datasets used and/or analyzed during the current study are available from the corresponding author on reasonable request.

\section{Authors' contribution}

G Y designed the study; Z C, P Z, Z Z, C G, C W, L L, H L, T G, D W performed the experiments; Z C and X D analyzed the data and interpreted the results of the experiments; $\mathrm{Z} C$ prepared the figures and wrote the manuscript; $L Z, X D$ and $L Y$ edited and revised the manuscript. All authors read and approved the final manuscript.

\section{Funding}

This study was supported by the National Natural Science Foundation of China $(81870548,81570721$, 82000809), the Social Development Project of Jiangsu Province (BE2018692), the Natural Science Foundation of Jiangsu Province, China (BK20191222), the Social Development Project of Zhenjiang City, Jiangsu Province (SH2019041) and the Scientific Research Projects of Jiangsu Health and Family Planning Commission (Y2018109).

\section{Acknowledgement}

All the authors of this manuscript have made substantial contributions to this work.

\section{References}

1. Pasquali R, Casanueva F, Haluzik M, van Hulsteijn L, Ledoux S, Monteiro MP, et al. European Society of Endocrinology Clinical Practice Guideline: Endocrine work-up in obesity. European Journal of Endocrinology.2020;182:G1-G32. 
2. Loiselle JJ, Yang G, Wu L. Hydrogen sulfide and hepatic lipid metabolism - a critical pairing for liver health.British Journal of Pharmacology.2018;177:757-768.

3. Nguyen $P$, Leray $V$, Diez M, Serisier S, Bloc'h JL, Siliart B, et al. Liver lipid metabolism.Journal of Animal Physiology and Animal Nutrition.2008;92:272-283.

4. Wang GX, Zhao XY, Meng ZX, Kern M, Dietrich A, Chen Z, et al. The brown fat-enriched secreted factor Nrg4 preserves metabolic homeostasis through attenuation of hepatic lipogenesis.Nature Medicine.2014;20:1436-1443.

5. Xiong X, Wang Q, Wang S, Zhang J, Liu T, Guo L, et al. Mapping the molecular signatures of dietinduced NASH and its regulation by the hepatokine Tsukushi.Molecular Metabolism.2019;20:128137.

6. Meex RCR, Watt MJ. Hepatokines: linking nonalcoholic fatty liver disease and insulin resistance.Nature Reviews Endocrinology.2017;13:509-520.

7. Stefan N, Häring HU. The role of hepatokines in metabolism.Nature Reviews Endocrinology.2013;9:144-152.

8. Watt MJ, Miotto PM, De Nardo W, Montgomery MK. The Liver as an Endocrine Organ-Linking NAFLD and Insulin Resistance.Endocrine Reviews.2019;40:1367-1393.

9. Arab JP, Arrese M, Trauner M. Recent Insights into the Pathogenesis of Nonalcoholic Fatty Liver Disease.Annual Review of Pathology: Mechanisms of Disease.2018;13:321-350.

10. Awazawa M, Gabel P, Tsaousidou E, Nolte H, Krüger M, Schmitz J, et al. A microRNA screen reveals that elevated hepatic ectodysplasin $A$ expression contributes to obesity-induced insulin resistance in skeletal muscle.Nature Medicine.2017;23:1466-1473.

11. Yang J, Zhou W, Zhu J, Wu Y, Xu L, Wang Y, et al. Circulating ectodysplasin A is a potential biomarker for nonalcoholic fatty liver disease.Clinica Chimica Acta.2019;499:134-141.

12. Donath MY, Burcelin R. GLP-1 Effects on Islets: Hormonal, Neuronal, or Paracrine?Diabetes Care.2013;36:S145-S148.

13. lepsen EW, Zhang J, Thomsen HS, Hansen EL, Hollensted M, Madsbad S, et al. Patients with Obesity Caused by Melanocortin-4 Receptor Mutations Can Be Treated with a Glucagon-like Peptide-1 Receptor Agonist.Cell Metabolism.2018;28:23-32.e3.

14. Xu W, Bi Y, Sun Z, Li J, Guo L, Yang T, et al. Comparison of the effects on glycaemic control and $\beta$-cell function in newly diagnosed type 2 diabetes patients of treatment with exenatide, insulin or pioglitazone: a multicentre randomized parallel-group trial (the CONFIDENCE study).Journal of Internal Medicine.2015;277:137-150.

15. Armstrong MJ, Hull D, Guo K, Barton D, Hazlehurst JM, Gathercole LL, et al. Glucagon-like peptide 1 decreases lipotoxicity in non-alcoholic steatohepatitis.Journal of Hepatology.2016;64:399-408.

16. Xu F, Li Z, Zheng X, Liu H, Liang H, Xu H, et al. SIRT1 Mediates the Effect of GLP-1 Receptor Agonist Exenatide on Ameliorating Hepatic Steatosis.Diabetes.2014;63:3637-3646. 
17. Nuffer WA, Trujillo JM. Liraglutide: A New Option for the Treatment of Obesity.Pharmacotherapy: The Journal of Human Pharmacology and Drug Therapy.2015;35:926-934.

18. Armstrong MJ, Gaunt P, Aithal GP, Barton D, Hull D, Parker R, et al. Liraglutide safety and efficacy in patients with non-alcoholic steatohepatitis (LEAN): a multicentre, double-blind, randomised, placebocontrolled phase 2 study. The Lancet.2016;387:679-690.

19. Anuurad E, Shiwaku K, Nogi A, Kitajima K, Enkhmaa B, Shimono K, et al. The new BMI criteria for asians by the regional office for the western pacific region of WHO are suitable for screening of overweight to prevent metabolic syndrome in elder Japanese workers.Journal of occupational health.2003;45:335-43.

20. Qian W, Zhu T, Tang B, Yu S, Hu H, Sun W, et al. Decreased circulating levels of oxytocin in obesity and newly diagnosed type 2 diabetic patients. The Journal of clinical endocrinology and metabolism.2014;99:4683-9.

21. Heo J, Yoon D, Yu J, Kim N, Yoo H, Seo J, et al. Melatonin improves insulin resistance and hepatic steatosis through attenuation of alpha-2-HS-glycoprotein.Journal of pineal research.2018;65:e12493.

22. Zhang C, Deng J, Liu D, Tuo X, Xiao L, Lai B, et al. Nuciferine ameliorates hepatic steatosis in high-fat diet/streptozocin-induced diabetic mice through a PPARa/PPARy coactivator-1a pathway.British journal of pharmacology.2018;175:4218-4228.

23. Jensen-Cody S, Potthoff M. Hepatokines and metabolism: Deciphering communication from the liver.Molecular metabolism.2021;44:101138.

24. Castro JP, Grune T, Speckmann B. The two faces of reactive oxygen species (ROS) in adipocyte function and dysfunction.Biological Chemistry.2016;397:709-724.

25. Saeedi P, Petersohn I, Salpea P, Malanda B, Karuranga S, Unwin N, et al. Global and regional diabetes prevalence estimates for 2019 and projections for 2030 and 2045: Results from the International Diabetes Federation Diabetes Atlas, 9th edition.Diabetes Research and Clinical Practice.2019;157.

26. Cuthbertson D, Irwin A, Gardner C, Daousi C, Purewal T, Furlong N, et al. Improved glycaemia correlates with liver fat reduction in obese, type 2 diabetes, patients given glucagon-like peptide-1 (GLP-1) receptor agonists.PloS one.2012;7:e50117.

27. Hira T, Pinyo J, Hara H. What Is GLP-1 Really Doing in Obesity?Trends in Endocrinology \& Metabolism.2020;31:71-80.

28. Gaballah HH, Zakaria SS, Mwafy SE, Tahoon NM, Ebeid AM. Mechanistic insights into the effects of quercetin and/or GLP-1 analogue liraglutide on high-fat diet/streptozotocin-induced type 2 diabetes in rats.Biomedicine \& Pharmacotherapy.2017;92:331-339.

29. Zhao L, Zhu C, Lu M, Chen C, Nie X, Abudukerimu B, et al. The key role of a glucagon-like peptide-1 receptor agonist in body fat redistribution.Journal of Endocrinology.2019;240:271-286.

30. Zizzari P, He R, Falk S, Bellocchio L, Allard C, Clark S, et al. CB1 and GLP-1 Receptors Cross-Talk Provides New Therapies for Obesity.Diabetes.2020.

31. Yang J, Ren J, Song J, Liu F, Wu C, Wang X, et al. Glucagon-like peptide 1 regulates adipogenesis in 3T3-L1 preadipocytes.International Journal of Molecular Medicine.2013;31:1429-1435. 
32. Yu X, Hao M, Liu Y, Ma X, Lin W, Xu Q, et al. Liraglutide ameliorates non-alcoholic steatohepatitis by inhibiting NLRP3 inflammasome and pyroptosis activation via mitophagy.European Journal of Pharmacology.2019;864.

\section{Tables}

Table 1. Clinical and biochemical characteristics in the study groups

\begin{tabular}{|c|c|c|c|c|}
\hline Parameters & Total $(\mathrm{n}=60)$ & $N W(n=30)$ & $\mathrm{OB}(\mathrm{n}=30)$ & $P$ value \\
\hline Sex,Males (\%) & 56.7 & 56.7 & 56.7 & 1 \\
\hline Age (years) & $45.83 \pm 12.28(26-71)$ & $47.13 \pm 11.63$ & $44.53 \pm 12.78$ & 0.417 \\
\hline Alcohol use (\%) & 18.3 & 20.0 & 16.7 & 0.739 \\
\hline Smoking (\%) & 36.7 & 47.7 & 26.7 & 0.184 \\
\hline BMI $\left(\mathrm{kg} / \mathrm{m}^{2}\right)$ & $25.17 \pm 3.85(17.61-34.02)$ & $22.08 \pm 1.82$ & $28.26 \pm 2.66$ & $<0.001$ \\
\hline WC $(\mathrm{cm})$ & $88.27 \pm 10.37(72-113)$ & $80.88 \pm 6.77$ & $95.66 \pm 7.76$ & $<0.001$ \\
\hline $\mathrm{HC}(\mathrm{cm})$ & $98.83 \pm 7.15(83-114.5)$ & $93.97 \pm 5.28$ & $103.70 \pm 5.21$ & $<0.001$ \\
\hline WHR & $0.89 \pm 0.06(0.80-1.05)$ & $0.86 \pm 0.05$ & $0.92 \pm 0.05$ & $<0.001$ \\
\hline SBP (mmHg) & $123.65 \pm 16.45(91-154)$ & $114.1 \pm 14.14$ & $133.2 \pm 12.71$ & $<0.001$ \\
\hline DBP (mmHg) & $75.85 \pm 14.73(48-110)$ & $69.07 \pm 12.95$ & $82.63 \pm 13.35$ & $<0.001$ \\
\hline HbA1c (\%) & $5.70 \pm 0.27(5.2-6.8)$ & $5.67 \pm 0.29$ & $5.73 \pm 0.25$ & 0.450 \\
\hline FPG $(\mathrm{mmol} / \mathrm{L})$ & $4.83 \pm 0.57(3.73-5.87)$ & $4.81 \pm 0.60$ & $4.94 \pm 0.53$ & 0.370 \\
\hline FINS $(\mu \mathrm{U} / \mathrm{ml})$ & $6.81 \pm 5.61(0.66-27.86)$ & $4.59 \pm 2.98$ & $9.04 \pm 6.70$ & 0.002 \\
\hline TG (mmol/L) & $1.61 \pm 0.79(0.56-4.13)$ & $1.29 \pm 0.54$ & $1.93 \pm 0.88$ & 0.001 \\
\hline $\mathrm{TC}(\mathrm{mmol} / \mathrm{L})$ & $4.9 \pm 0.81(3.32-6.62)$ & $4.78 \pm 0.77$ & $5.02 \pm 0.83$ & 0.247 \\
\hline HDL-C (mmol/L) & $1.35 \pm 0.36(0.66-2.48)$ & $1.49 \pm 0.39$ & $1.22 \pm 0.27$ & 0.002 \\
\hline LDL-C (mmol/L) & $2.81 \pm 0.72(1.42-4.45)$ & $2.63 \pm 0.68$ & $2.99 \pm 0.72$ & 0.052 \\
\hline ALT (U/L) & $25.73 \pm 15.60(5-75.7)$ & $20.3 \pm 14.71$ & $31.13 \pm 18.80$ & 0.016 \\
\hline AST (U/L) & $20.85 \pm 12.32(9.9-86.5)$ & $20.87 \pm 15.16$ & $20.83 \pm 8.88$ & 0.991 \\
\hline$\gamma$-GT $(\mathrm{U} / \mathrm{L})$ & $29.63 \pm 24.91(10-176)$ & $25.78 \pm 29.97$ & $33.48 \pm 18.27$ & 0.234 \\
\hline HOMA-IR & $1.53 \pm 1.33(0.12-6.5)$ & $1.04 \pm 0.72$ & $2.03 \pm 1.61$ & 0.003 \\
\hline HOMA- $\beta$ & $111.45 \pm 87.62(18.41-413.6)$ & $87.71 \pm 80.31$ & $135.18 \pm 89.47$ & 0.035 \\
\hline EDA $(\mathrm{pg} / \mathrm{ml})$ & $239.07 \pm 81.40(117.22-433.02)$ & $215.03 \pm 77.31$ & $263.12 \pm 76.8$ & $<0.001$ \\
\hline
\end{tabular}

Data are presented as means \pm SD and percentages. 
Abbreviations: BMI, body mass index; WC, waist circumference; HC, hip circumference; WHR, waist-to-hip ratio; SBP, systolic blood pressure; DBP, diastolic blood pressure; HbA1c, glycosylated hemoglobin c; FPG, fasting plasma glucose; FINS, fasting plasma insulin; TG, triglyceride; TC, total cholesterol; LDL-C, low-density lipoprotein cholesterol; HDL-C, highdensity lipoprotein cholesterol; ALT, alanine aminotransferase; AST, aspartate aminotransferase; GGT, g-glutamyl transpeptidase; HOMA-IR, homeostasis model assessmentinsulin resistance index; HOMA- $\beta$, homeostasis model assessment- $\beta$.

Table 2. Clinical and Biochemical Characteristics of the Study Subjects According to the Tertiles of Ectodysplasin A 


\begin{tabular}{|c|c|c|c|c|}
\hline Parameters & $\begin{array}{l}\text { Lower Tertile } \\
\qquad(\mathrm{n}=20)\end{array}$ & $\begin{array}{l}\text { Middle Tertile } \\
\qquad(\mathrm{n}=20)\end{array}$ & $\begin{array}{l}\text { Upper Tertile } \\
\qquad(\mathrm{n}=20)\end{array}$ & $P$ value \\
\hline EDA $(\mathrm{pg} / \mathrm{ml})$ & $<184.82$ & $184.82-259.81^{\mathrm{b}}$ & $>259.81^{b d}$ & $<0.001$ \\
\hline Sex, Males (\%) & 30.0 & $70.0^{\mathrm{a}}$ & $70.0^{\mathrm{a}}$ & 0.013 \\
\hline Age (years) & $49.45 \pm 12.40$ & $43.8 \pm 12.00$ & $44.25 \pm 12.24$ & 0.272 \\
\hline Alcohol use (\%) & 20.0 & 15.0 & 20.0 & 0.895 \\
\hline Smoking (\%) & 25.0 & 35.0 & 50.0 & 0.256 \\
\hline BMI $\left(\mathrm{kg} / \mathrm{m}^{2}\right)$ & $22.74 \pm 2.77$ & $26.46 \pm 3.95^{b}$ & $26.31 \pm 3.66^{b}$ & 0.002 \\
\hline $\mathrm{WC}(\mathrm{cm})$ & $81.58 \pm 8.18$ & $90.63 \pm 9.73^{b}$ & $92.60 \pm 9.99^{\mathrm{b}}$ & 0.001 \\
\hline $\mathrm{HC}(\mathrm{cm})$ & $94.54 \pm 6.07$ & $100.35 \pm 6.97^{b}$ & $101.605 \pm 6.58^{b}$ & 0.003 \\
\hline WHR & $0.86 \pm 0.05$ & $0.90 \pm 0.06^{\mathrm{a}}$ & $0.91 \pm 0.06^{b}$ & 0.021 \\
\hline SBP (mmHg) & $115 \pm 11.74$ & $126.7 \pm 18.32^{\mathrm{a}}$ & $129.25 \pm 15.65^{b}$ & 0.012 \\
\hline DBP (mmHg) & $70.0 \pm 11.00$ & $77.5 \pm 18.02$ & $79.15 \pm 13.70$ & 0.174 \\
\hline HbA1c (\%) & $5.72 \pm 0.34$ & $5.74 \pm 0.21$ & $5.64 \pm 0.25$ & 0.459 \\
\hline FPG (mmol/L) & $4.86 \pm 0.60$ & $4.87 \pm 0.60$ & $4.87 \pm 0.53$ & 0.992 \\
\hline FINS $(\mu \mathrm{U} / \mathrm{ml})$ & $5.98 \pm 4.14$ & $6.28 \pm 5.46$ & $8.18 \pm 6.91$ & 0.412 \\
\hline $\mathrm{TG}(\mathrm{mmol} / \mathrm{L})$ & $1.45 \pm 0.87$ & $1.61 \pm 0.82$ & $1.76 \pm 0.68$ & 0.465 \\
\hline $\mathrm{TC}(\mathrm{mmol} / \mathrm{L})$ & $5.01 \pm 0.88$ & $4.90 \pm 0.93$ & $4.80 \pm 0.59$ & 0.711 \\
\hline HDL-C (mmol/L) & $1.51 \pm 0.39$ & $1.30 \pm 0.27$ & $1.24 \pm 0.36^{\mathrm{a}}$ & 0.042 \\
\hline LDL-C (mmol/L) & $2.75 \pm 0.76$ & $2.89 \pm 0.84$ & $2.80 \pm 0.56$ & 0.819 \\
\hline $\operatorname{ALT}(\mathrm{U} / \mathrm{L})$ & $27.41 \pm 19.58$ & $25.55 \pm 14.30$ & $24.22 \pm 19.22$ & 0.851 \\
\hline AST (U/L) & $25.51 \pm 19.58$ & $20.13 \pm 6.32$ & $16.93 \pm 2.87$ & 0.082 \\
\hline$\gamma$-GT (U/L) & $33.88 \pm 38.70$ & $26.70 \pm 13.58$ & $28.33 \pm 14.68$ & 0.641 \\
\hline HOMA-IR & $1.38 \pm 1.01$ & $1.39 \pm 1.28$ & $1.83 \pm 1.65$ & 0.497 \\
\hline HOMA- $\beta$ & $105.31 \pm 89.26$ & $100.20 \pm 74.80$ & $128.83 \pm 98.93$ & 0.553 \\
\hline
\end{tabular}

a $\mathrm{P}<0.05$ compared with lower tertile.

b $\mathrm{P}<0.01$ compared with lower tertile.

${ }^{\mathrm{C}} \mathrm{P}<0.05$ compared with middle tertile.

$\mathrm{d} \mathrm{P}<0.01$ compared with middle tertile. 
Data are presented as means \pm SD and percentages.

Abbreviations: BMI, body mass index; WC, waist circumference; HC, hip circumference; WHR, waist-to-hip ratio; SBP, systolic blood pressure; DBP, diastolic blood pressure; HbA1c, glycosylated hemoglobin c; FPG, fasting plasma glucose; FINS, fasting plasma insulin; TG, triglyceride; TC, total cholesterol; LDL-C, low-density lipoprotein cholesterol; HDL-C, highdensity lipoprotein cholesterol; ALT, alanine aminotransferase; AST, aspartate aminotransferase; GGT, g-glutamyl transpeptidase; HOMA-IR, homeostasis model assessmentinsulin resistance index; HOMA- $\beta$, homeostasis model assessment- $\beta$.

Table 3. Stepwise Multiple Linear Regression Analysis with EDA as the Dependent Variable ${ }^{a}$

\begin{tabular}{llll}
\hline Independent Variable & Regression Coefficient (SE) & 95\%CI & P value \\
\hline BMI & $7.608(0.360)$ & $2.424-12.793$ & 0.005 \\
\hline
\end{tabular}

Abbreviation: CI, confidence interval.

a The following independent variables were considered for the model: BMI, WHR, SBP, DBP, FBG, FIns, HbA1c, TG, TC, HDL-C, LDL-C, ALT, AST, $\gamma$-GT, HOMA-IR and HOMA- $\beta$. Only the variables that had a $\mathrm{P}<0.05$ were considered in the final fitted model.

\section{Figures}



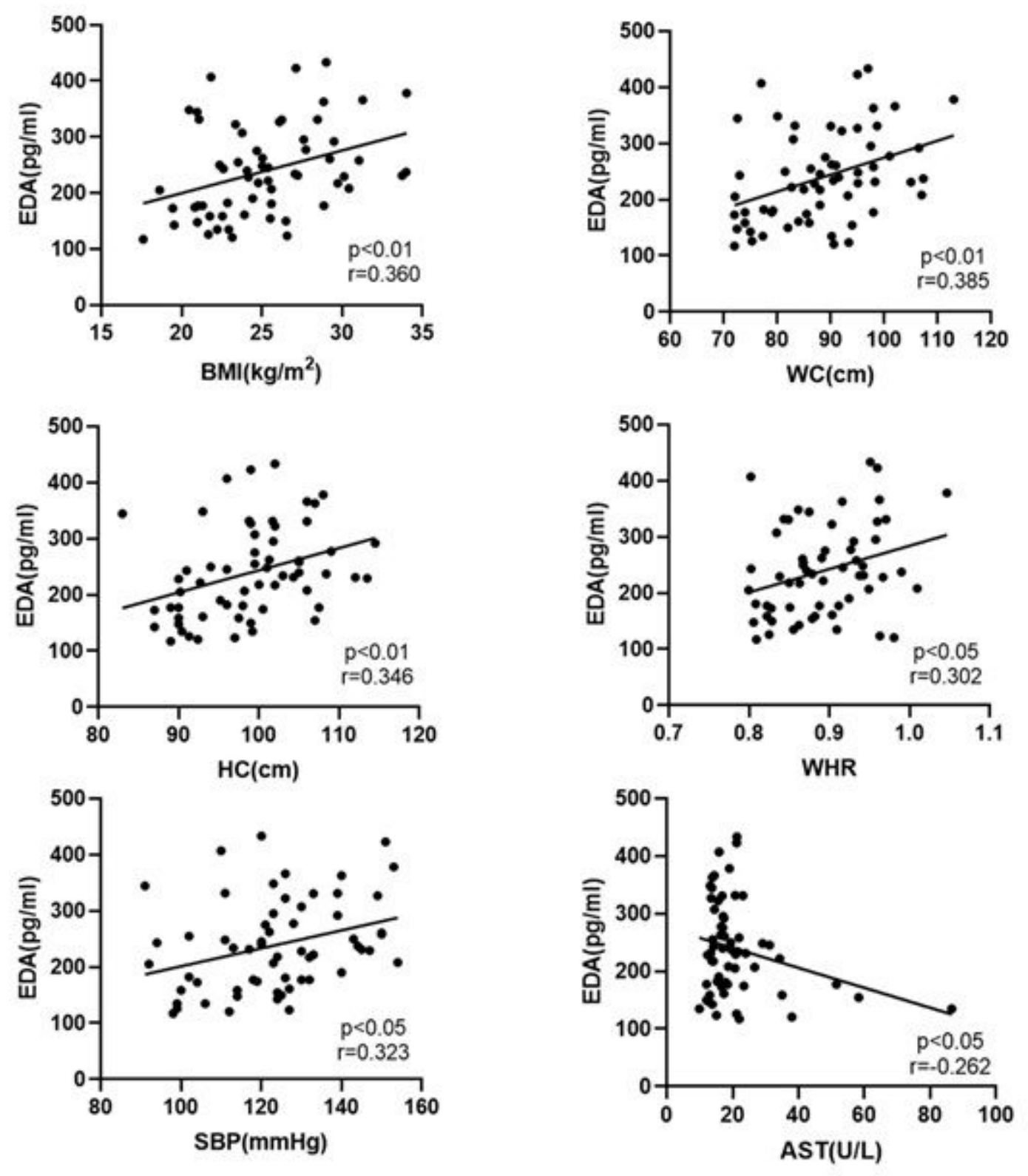

Figure 1

Correlations between serum EDA levels and metabolic parameters such as body mass index (BMI), waist circumference (WC), hip circumference (HC), waist-to-hip ratio (WHR), systolic blood pressure (SBP) and aspartate aminotransferase (AST). 
A
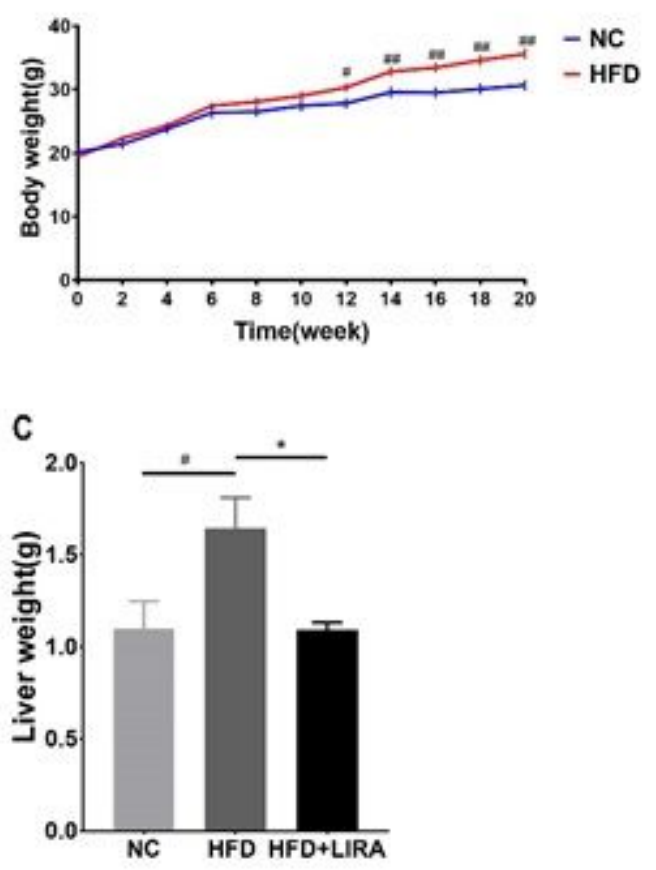

B

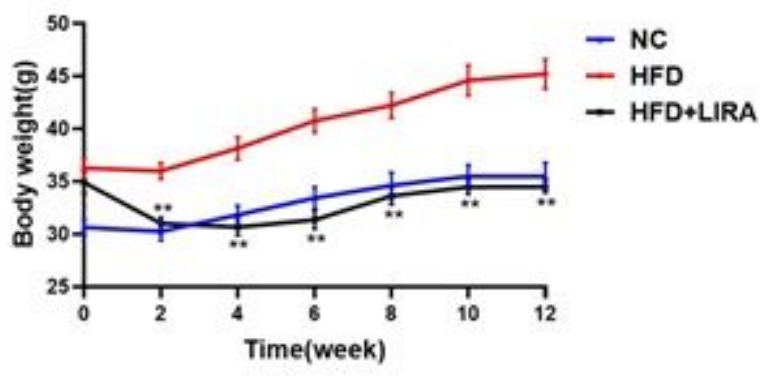

\section{Figure 2}

Changes in body weight and liver weight among mice in three groups. Mice in HFD+LIRA group were received daily subcutaneous injections with liraglutide $(0.2 \mathrm{mg} / \mathrm{kg}$ daily) and HFD and NC mice were given the same volume of saline during the same period (A) Body weight before liraglutide treatment $(n=5)$. (B) Body weight after liraglutide treatment $(n=5)$. (C) Liver weight of mice $(n=5)$. P Values are mean \pm S.E.M \#P<0.05, \#\#P<0.01: vs HFD mice + NC mice. ${ }^{*} P<0.05,{ }^{*} * \mathrm{P}<0.01$ : vs HFD mice + HFD+LIRA mice.

A

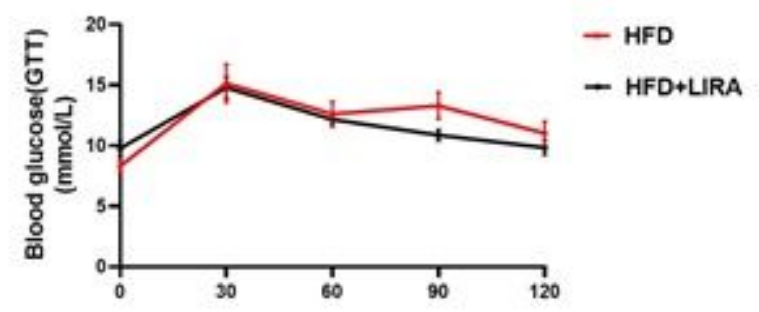

c

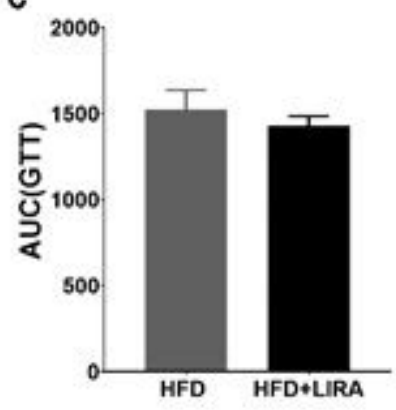

B

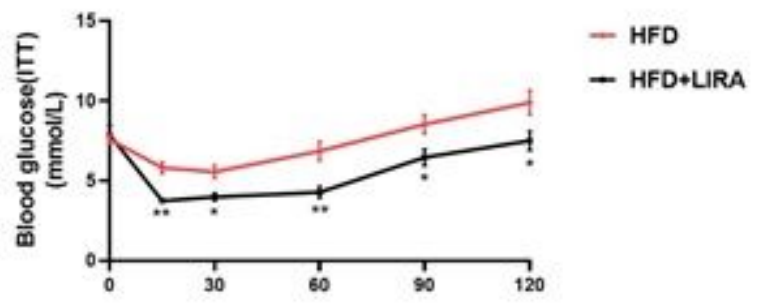

D

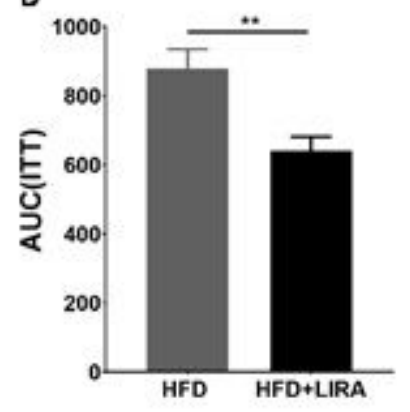




\section{Figure 3}

Dynamic changes in blood glucose levels after liraglutide treatment in HFD-induced obese mice. Mice were starved for $4 \mathrm{~h}$ and $16 \mathrm{~h}$, respectively, before an intraperitoneal injection of insulin $(0.75 \mathrm{units} / \mathrm{kg}$ of body weight) or glucose (2.0 g/ $\mathrm{kg}$ of body weight). (A) Glucose tolerance test and (B)insulin tolerance test after liraglutide treatment $(n=5)$. (C) Area under the curve of GTT and (D)ITT $(n=5)$. *P<0.05, $\star * P<0.01$ : vs HFD mice + HFD+LIRA mice.

A
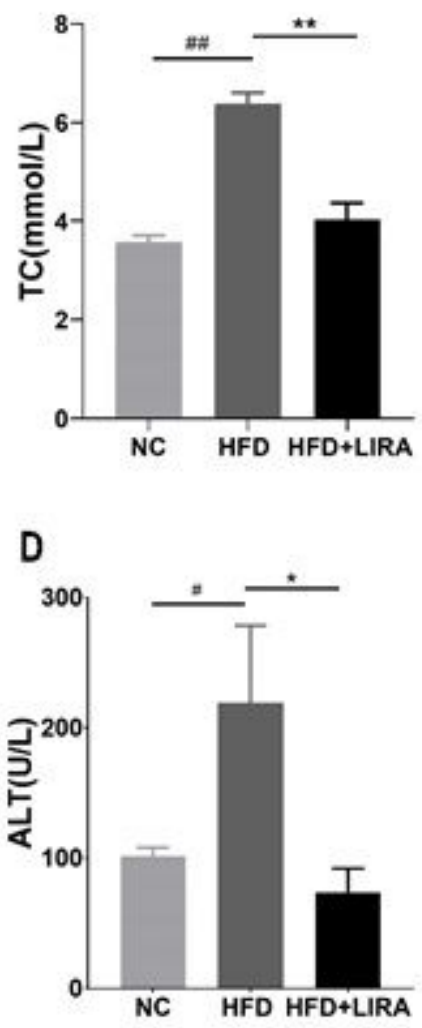

B
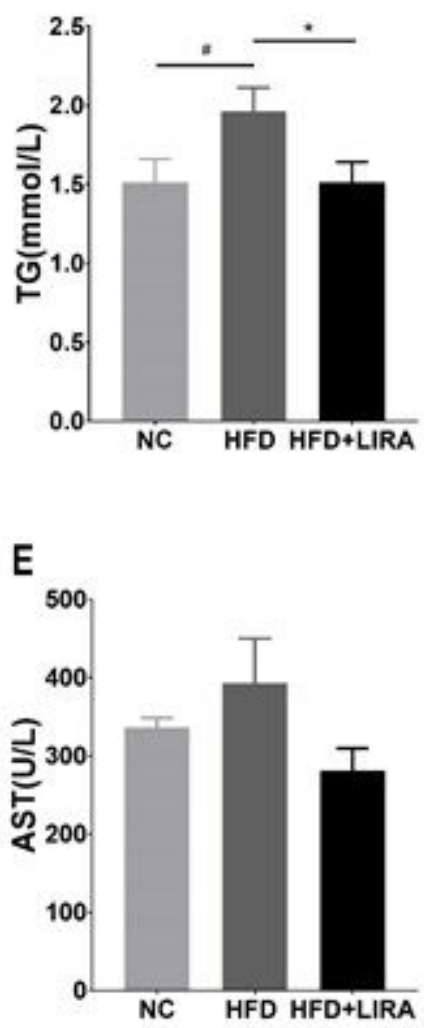

C

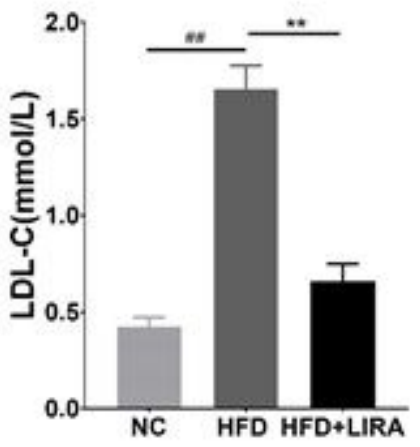

\section{Figure 4}

Effects of liraglutide on hepatic steatosis and blood lipid levels in HFD-induced obese mice. (A) Total cholesterol (TC). (B) Triglyceride (TG). (C) Low density lipoprotein cholesterol (LDL-C). (D) Alanine aminotransferase (ALT). (E) Aspartate aminotransferase (AST) in NC mice and obese mice $(n=5)$. $\# P<0.05, \# \# P<0.01$ : vs HFD mice $+N C$ mice. ${ }^{*} P<0.05,{ }^{*} P<0.01$ : vs HFD mice + HFD+LIRA mice. 

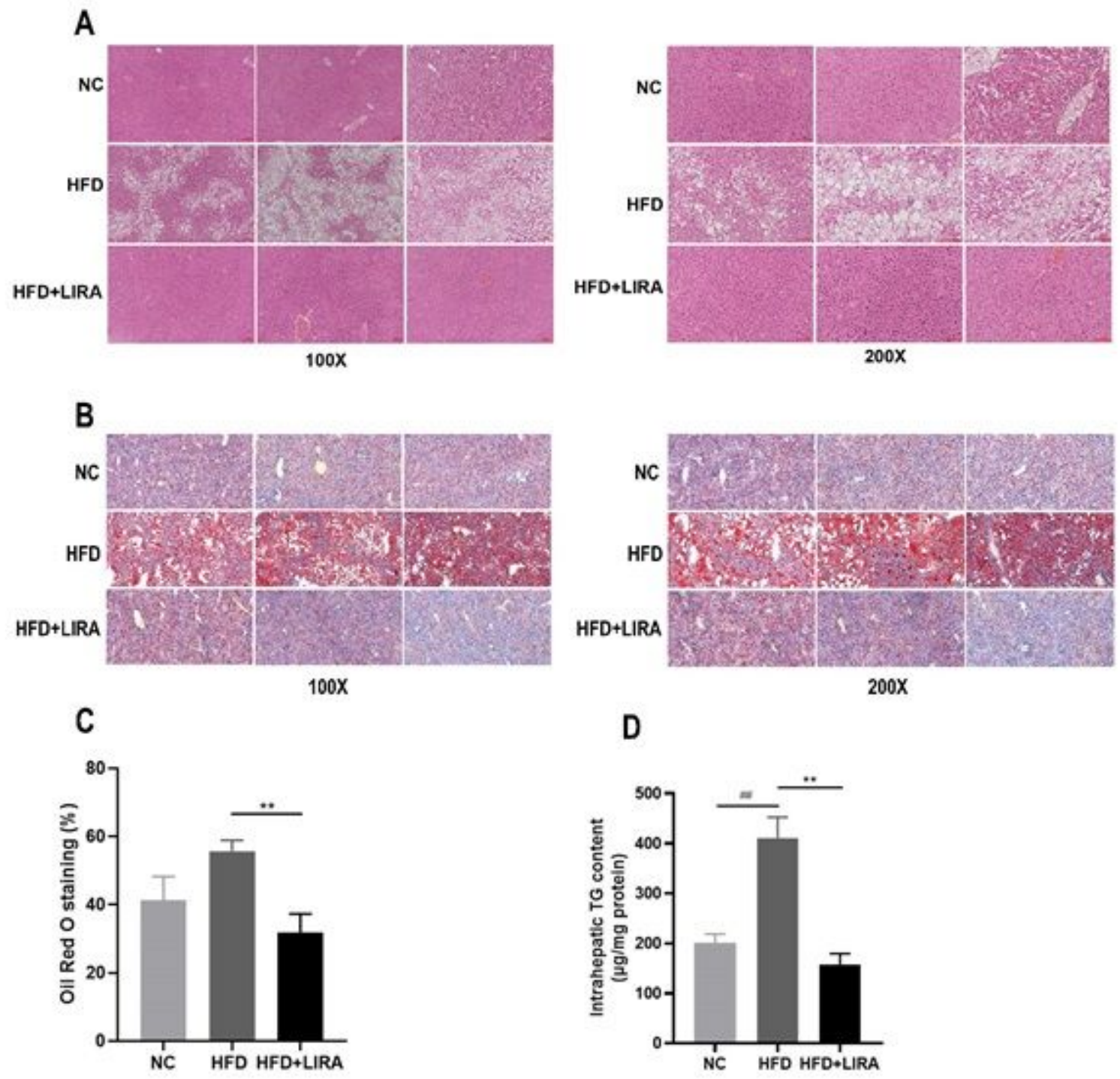

Figure 5

Liraglutide improved hepatic steatosis. (A) HE staining of liver tissues (magnification $\times 100$ and $\times 200$ ). (B) Oil Red 0 staining of liver tissues (magnification $\times 100$ and $\times 200$ ). (C) Area ratios of oil red 0 staining $(n=5)$. (D) Intrahepatic triglyceride $(T G)$ content $(n=5)$. 
A

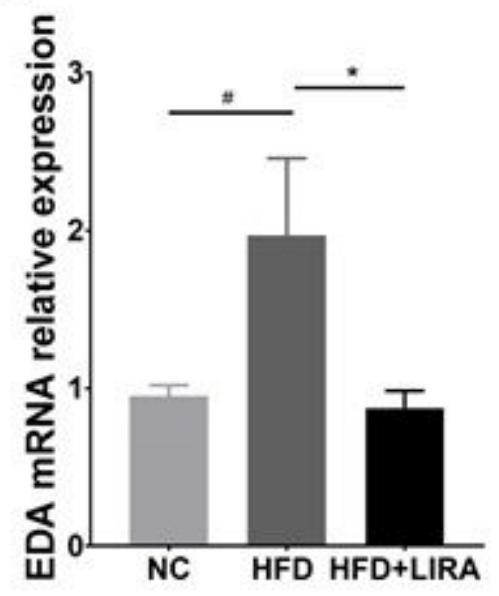

B
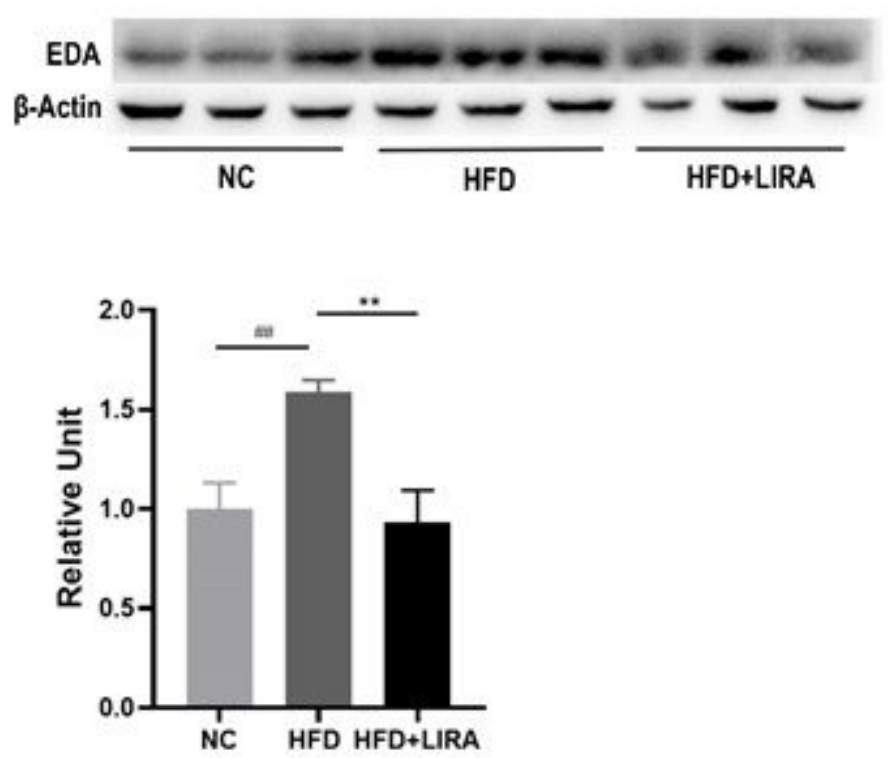

Figure 6

The expression of EDA in liver tissues after liraglutide treatment. (A) mRNA expression of EDA in HFDinduced obese mice $(n=5)$. (B) Protein expression of EDA in HFD-induced obese mice $(n=5)$. \#P<0.05, $\# \# P<0.01$ : vs HFD mice + NC mice. ${ }^{\star} P<0.05$, ${ }^{\star *} P<0.01$ : vs HFD mice + HFD+LIRA mice.

A

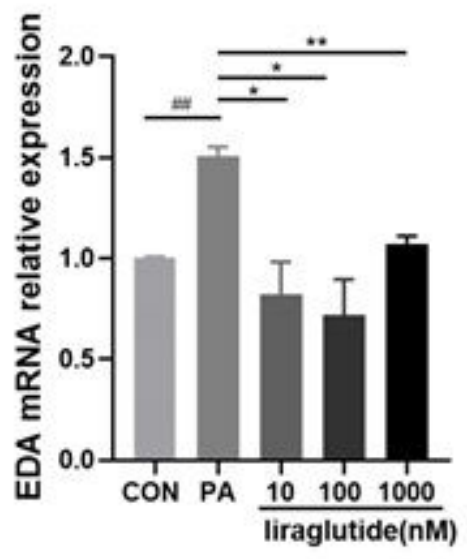

B
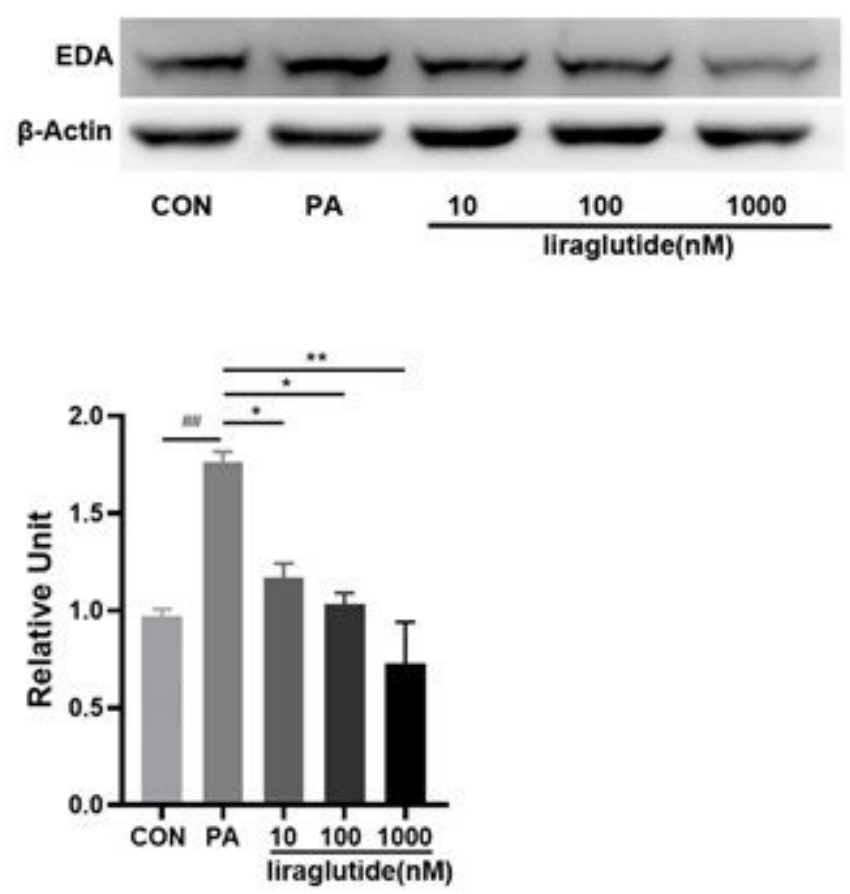

Figure 7 
The expression of EDA in AML12 cells after liraglutide treatment. (A) mRNA expression of EDA in PAinduced AML12 cells with different concentrations $(10,100,1000 \mathrm{nM})$. (B) Protein expression of EDA in PAinduced AML12 cells with different concentrations $(10,100,1000 \mathrm{nM})$. \#\#P<0.01: vs HFD mice + NC mice. ${ }^{\star} P<0.05,{ }^{\star *} P<0.01$ : vs HFD mice + HFD+LIRA mice. 